\title{
NONLINEAR INEQUALITIES AND RELATED FIXED POINT PROBLEMS
}

\author{
MUHAMMAD NAZAM, ESKANDAR AMEER, \\ MOHAMMAD MURSALEEN* AND ÖZLEM ACAR
}

\begin{abstract}
In this paper, we introduce a nonlinear inequality based on four self-mappings. We give necessary conditions which ensure the existence of a common fixed point of four selfmappings satisfying said inequality defined in $S$-metric spaces. A common fixed point problem is discussed. We set up an example to elucidate our main result. Moreover, the existence of a common solution to a system of four integral equations is shown by application of main result.
\end{abstract}

Mathematics subject classification (2020): 47H10, 39B62, 26E05.

Keywords and phrases: Nonlinear inequalities, common fixed point problems, complete $S$-metric space, application.

\section{REFERENCES}

[1] O. ACAR, I. ALtUn, Multivalued $F$-contractive mappings with a graph and some fixed point results, Publ. Math. Debrecen 88 (2016), 305-317.

[2] M. U. Ali, T. KAMRAN, Multivalued F-contractions and related fixed point theorems with an application, Filomat 30 (14) (2016), 3779-3793.

[3] M. ASADI, P. SAlimi, Some Fixed point and common fixed point theorems for G-metric spaces, Nonlinear Funct. Anal. Appl., 21 (3) (2016), 523-530.

[4] S. BANACH, Sur les opérations dans les ensembles abstraits et leur application aux equations itegrales, Fund. Math. 3 (1922), 133-181.

[5] S. CZerwik, Nonlinear set-valued contraction mappings in b-metric spaces, Atti Semin. Mat. Fis. Univ. Modena. 46 (2) (1998), 263-276.

[6] S. CzerwiK, Contraction mappings in b-metric spaces, Acta Math. Inf. Univ. Ostrav 1 (1993), 5-11.

[7] B. C. DhaGe, Generalized metric space and mapping with fixed point, Bulletin of the Calcutta Mathematical Society 84 (1992), 329-336.

[8] B. C. Dhage, On generalized metric spaces and topological structure II, Pure and Applied Mathematika Sciences 40 (1994), 37-41.

[9] G. DurmaZ, G. Minak, I. Altun, Fixed points of ordered F-contractions, Hacettepe Journal of Mathematics and Statistics 45 (1) (2016), 15-21.

[10] S. GAhleR, 2-metrische Raume und ihre topologische Struktur, Mathematische Nachrichten 26 (1963), 115-148.

[11] S. Gahler, Zur geometric 2-metriche raume, Revue Roumaine de Mathematiques Pures et Appliquees 11 (1966), 664-669.

[12] A. Javaheri, S. Sedghi, H. G. Hyun, Common fixed point theorems for two mappings in S-metric spaces, Nonlinear Funct. Anal. Appl., 24 (2) (2019), 417-425.

[13] Z. Mustafa, B. Sims, A new approach to generalized metric spaces, J. Nonlinear Convex Anal. 7 (2006), 289-297.

[14] M. NAZAm, N. Hussain, A. Huss Ain, M. Arshad, Fixed point theorems for weakly admissible pair of F-contractions with application, Nonlinear Analysis: Modelling and Control 24 (6) (2019), 898-918.

[15] H. PiRI, P. Kumam, Some fixed point theorems concerning F-contraction in complete metric spaces, Fixed Point Theory Appl. 2014 (2014), 11 pages. 
[16] M. M. Rezaee, S. Sedghi, K. S. KIM, Coupled common fixed point results in ordered S-metric spaces, Nonlinear Func. Anal. Appl., 23 (3) (2018), 595-612.

[17] S. Sedghi, N. Shobe, A. Aliouche, A generalization of fixed point theorem in S-metric spaces, Mat. Vesnik 64 (2012), 258-266.

[18] S. Sedghi, N. Shobe, H. Zhou, A common fixed point theorem in $D^{*}$-metric spaces, Fixed Point Theory Appl. Vol. 2007, Article ID 27906 (2007), 13 pages.

[19] S. Sedghi, A. Gholidahneh, K. P. R. Rao, Common fixed point of two R-weakly commuting mappings in $S_{b}$-metric spaces, Math. Sci. Lett. 6 (3) (2017), 249-253.

[20] D. Singh, V. Joshi, J. K. KIM, Existence of solution to Bessel-type boundary value problem via G-l cyclic F-contractive mappings with graphical verification, Nonlinear Funct. Anal. Appl., 23 (2) (2018), 205-224.

[21] D. WARDOWS KI, Fixed points of a new type of contractive mappings in complete metric spaces, Fixed Point Theory Appl. 2012, (2012): 6 pages.

[22] M. Younis, D. Singh, D. Gopal, A. Goyal, M. S. Rathore, On applications of generalized F-contraction to differential equations, Nonlinear Func. Anal. Appl., 24 (1) (2019), 155-174. 